A. Pramesh Rao, G. Swarup and Gopal-Krishna, eds.

\title{
Populations of Hydrogen-like Atoms or Ions and Radio Recombination Lines (RRL's) Interpretation.
}

\author{
N.I. Rovenskaya \\ Institute of Radio Astronomy,Krasnoznamennaya 4,Kharkov, Ukraine
}

\begin{abstract}
The problem of non-LTE populations has been considered in terms of the departure coefficients $\frac{\partial b_{n}}{\partial n}$ as functions of the kinetic temperature $T_{e}$, the electron density $N_{e}$, the continuum radiation flow $I_{c}$ and the ratios of $I_{H n \alpha}, I_{H n \beta}, I_{H n \delta}$ and $I_{H n \varepsilon}$ (the line radiation flows). The ratio of $I_{H n \alpha} / I_{H n \beta}$ are sensitive to the thermal radiation from HII regions. Characterized by the relation of $\frac{\partial^{2} b_{n}}{\partial n^{2}}>0$, the populations are shown to be inhabited radiatively.
\end{abstract}

\section{Introduction}

The numerical programmes by Brocklehurst \& Salem (1979) provide an exact solution to the balance equations for hydrogen-like, highly excited atoms in the model, constructed by Seaton (1964) and supplemented with the details by Dyson (1967), Shaver(1975) and Hoang-Binh (1986). RRLs are observed in the centimetre and millimetre wave bands (Cersosimo \& Magnani,1990; Gordon \& Walmsley,1990; Anantharamaiah et al 1988; Hoang-Binh et al 1985). The analytical methods have been stimulated by experiments at the low-frequency range. The low-frequency lines are formed between the levels with numbers $n=$ $308-750$ and they are observed by Sorochenko et al (1984), Konovalenko (1984) and Anantharamaiah et al (1992) with the instruments of UTR-2 (Kharkov), DKR-1000 (Pushino) and NRAO. The balance equations are analytically solved for the superhigh levels of $n=600-1000$ by Vainstein et al (1979), Beigman (1987) and Rovenskaya (1992). Owing to the interaction of hydrogen-like atoms and ions with this thermal radiation of the radio sources with flat Rayleigh-Jeans spectrum, the ratio of RRL flows can be estimated as follows $I_{H n \alpha} / I_{H n \beta} \sim$ $(n \beta / n \alpha)^{3.3}$ and $\frac{\partial^{2} b_{n \beta}}{\partial n \beta^{2}}>0$, where $n \alpha, n \beta$ are the numbers of RRLs. Owing to atomic collisions with electrons the analogous magnitude takes the form $I_{H n \delta} / I_{H n \varepsilon} \sim(n \varepsilon / n \delta)^{3}, \frac{\partial^{2} b_{n \delta}}{\partial n \delta^{2}}<0$ and $n \delta<n \varepsilon$. The well-known numerical programmes by Brocklehurst \& Salem (1979) are complemented with the $\frac{\partial b_{n}}{\partial n}$ factors for heavy ions of $\mathrm{He}, \mathrm{C}$ and $\mathrm{Fe}$.

The temperature and the density of HII region are calculated as functions of the kinetic coefficients.

$$
D^{c} n^{7}>D^{R} n^{7.3}, D^{B B} n^{3}
$$

where $D^{c}$ is the collisional kinetic coefficient, $D^{R}$ and $D^{B B}$ are the radiative kinetic coefficients. 
The temperature is found using the RRL radiation ratio as follows $I_{H n}^{\text {peak }} / I_{c}$, where $I_{H n}^{\text {peak }}$ is the RRL peak intensity, $I_{c}$ is the continuum intensity at the same frequency.

$$
T_{e}=\nu^{2}\left[\frac{\left(1-\beta_{n}\right) b_{n}}{T_{e}}\right] \frac{I_{c}^{2}}{2 I_{H n}^{p e a k}} \cdot \frac{1.9 \cdot 10^{2}}{(\Delta n)^{1.85}} \cdot \frac{1}{3.14 \cdot 10^{-2} \ln \left(10^{6} / 20.18 \nu\right)},
$$

where $\nu$ is the radiation frequency $(\mathrm{GHz}), \frac{\left(1-\beta_{n}\right) b_{n}}{T_{e}}$ is the coefficient as function of the Seaton matrix $A_{n 1}$ and the quantum numbers $n 1$ and $n ; I_{c}$ is the continuum radiation intensity at the frequency $\nu ; I_{H n}^{p e a k}$ is the RRL radiation intensity; $\Delta n$ describes the high order line number in the following way $\Delta n=1,2,3,4$ and 5 for $H_{n \alpha}, H_{n \beta}, H_{n \gamma}, H_{n \delta}$ and $H_{n \varepsilon}$. If the RRL number $n$ is more than $n 1$, term (1) is changed for solution in the form

$$
\frac{\left(1-\beta_{n}\right) b_{n}}{T_{e}}=\frac{n^{2}(1-3 / n)}{1.58 \cdot 10^{5}} \frac{\exp \left(-(n 1 / n)^{2}\right)}{\left(A_{n 1}+\pi\right)(n / n 1)^{5}},
$$

where $A_{n 1}$ and $n 1$ are the Seaton matrix and the quantum number determined from relations $(3)$.

The magnitude of the number $n 1$ can be found experimentally by comparison of high orders RRL intensities at the nearest frequencies. Using formula (3) the number $n 1$ is found fron the following relation

$$
\frac{I_{H m 1}}{I_{H m 2}}\left(\frac{\Delta m 1}{\Delta m 2}\right)^{1.85}\left(\frac{m 1}{m 2}\right)^{3}=\exp \left[-(n 1)^{2}\left[\frac{1}{m 1}-\frac{1}{m 2}\right]\right],
$$

where $m 1, m 1+\Delta m 1$ are the numbers corresponded to the two atomic levels of $\mathrm{RRL} ; I_{H m 1}$ is the hydrogenic RRL intensity as function of the number $m 1$.

According to formula (4) the number $n 1$ is determined as function of the RRL radiation intensity. When the number $n 1$ is found it is easy to calculate the density $N_{e}$ by the method of the equation as following

$$
\left.\frac{d}{d n}\left(1-\beta_{n}\right)\right|_{n=n 1}=0 \text {. }
$$

The collisional coefficient equals $D_{n}^{c}=1.88 \cdot 10^{-14} N_{e} T_{e}^{-1 / 2} n^{7}$.

The density $N_{e}$ is determined from

$$
N_{e}=\frac{\left(A_{n 1}+\pi\right)}{1.88 \cdot 10^{-14} n 1^{7}} T_{e}^{1 / 2}
$$

For some analysis the plasma temperature and the density are calculated by the ratio of $H_{56 \delta}$ and $H_{60 \varepsilon}$ intensities when the RRL numbers $n$ is $n \leq n 1$. For these estimations the experimental data is chosen following the work by Gordon \& Walmsley (1990). These are HII regions of W3, W49, DR21, which are studied.

\section{References}

Rovenskaya N.I.,1999, Astrophys.Space Sci., 259, 1 